\title{
Optimal Timing For The Sale Of An Indivisible Asset With Jumps: A Numerical Approach
}

Bader Alhashel, Kuwait University, Kuwait

\begin{abstract}
This paper examines the situation in which a utility maximizing agent holds a portfolio composed of a Real Asset and a proportion of a market portfolio. The Real Asset is indivisible, and its sale is irreversible and results in a one-time payment. The question this paper attempts to answer is, "When is the optimal time the agent should sell this Real Asset?". In other words, at what proportion of the agent's wealth should the Real Asset be sold. This paper extends the work of Evans et al. (2008) through adding a jump process to the stochastic process of the Real Asset to better capture its idiosyncratic risks. The results can be summarized into three strategies: i) sell immediately, ii) sell at a certain proportion of wealth, and iii) never sell the asset. Furthermore, we have found evidence showing the significance of the addition of the jump process. This addition affects the agent's optimal path by pushing him to hold on to the Real Asset at smaller fractions of his wealth when compared to the original version of no jumps.
\end{abstract}

Keywords: Real Assets; Real Options; Incomplete Markets; Jump-Diffusion Process; Numerical

\section{INTRODUCTION}



vans, Henderson, and Hobson (2008), hereinafter referred to as EHH, attempts to find a solution for the optimal timing for an indivisible asset sale. In this problem, the asset is indivisible, not traded dynamically, and the sale is irreversible and results in a one-time payoff. Examples of this are the selling of a plant or a piece of land or the selling of mining rights. Institutions and individuals confront these examples and numerous others on a continuous basis. Such managerial decisions on investing and abandoning are usually treated as Real Options. However, the difference here, with respect to most of the literature, is that it is not assumed that the markets are complete, nor is the existence of a replicating portfolio assumed. The ultimate question their paper attempts to answer is when an agent should sell this Real Asset.

We extend EHH's model through attempting to better model the behavior of the Real Asset. Given the features of the Real Asset, an agent cannot completely hedge the risks associated with it, thereby exposing him to the idiosyncratic risk of the asset. Idiosyncratic risk stems from the possible occurrence of asset-specific events that significantly reduce its value (e.g., important news events). To better capture this risk in the stochastic behavior of the asset, we follow the extant literature in adding a jump process to its Brownian motion. Modeling the evolution of the Real Asset value as a jump-diffusion process better captures the asset's motions and the occurrence of jumps in its returns (Ball and Torous, 1985; Lee and Mykland, 2008; Maheu and McCurdy, 2004; Press, 1967).

Jumps have been increasingly incorporated into the pricing of various financial assets since the seminal work of Merton (1976) in enhancing the Black-Scholes option pricing formula to capture such a stochastic process. Several papers have incorporated the jump-diffusion process to improve the pricing of options and better capture the behavior of the underlying asset (e.g., Andersen and Andresen, 2000; Cont and Voltchkova, 2005; Cox and Ross, 1976; Duffie, Pan, and Singleton, 2000; Kou, 2002; Kou and Wang, 2004). Additional incorporations of a jump process have also been used in various other financial assets such as lookback options (Kou and Wang, 2003), barrier options (Cai and Kou, 2011), exchange rate American options (Bates, 1996) and interest rates (Das, 2002). 
We find that the jump process does affect the optimal behavior of the agent holding the Real Asset. Furthermore, as the idiosyncratic risk increases, the agent becomes more reluctant to hold on to the Real Asset, driving him to dispose of it earlier than when compared to the case of no jumps.

The paper contributes to the real options literature. Managers can make decisions that alter the cash flows generated by the Real Assets they hold even after the asset has been purchased. Such decisions are termed real options and examples of this would include the option to expand production and the option to abandon the Real Asset before its initially planned maturity ${ }^{1}$. The problem we concentrate on in this paper is determining the optimal time to sell the Real Asset. The real options literature, starting with McDonald and Siegel (1986), assumes that markets are complete and that there exists a replicating portfolio. A strand within this literature has departed from these assumptions and assumed that markets are incomplete and that there is no replicating portfolio (e.g., EHH; Henderson (2007); Smith and Nau (1995)). More specifically, EHH extends the literature by finding a closed-form solution to the optimal exercise criterion and the value of the option to sell. We contribute to the literature by further developing the EHH model to better capture the behavior of the asset's returns and risks through modeling it as a jump-diffusion process.

In the next section we present the model. Section 3 contains the numerical solution and Section 4 concludes this paper.

\section{THE MODEL}

The model follows that of EHH. The only difference is the addition of a jump process, $\xi d q$, to the Brownian motion of the Real Asset in Equation 1.

Suppose that a utility maximizing agent has one indivisible Real Asset whose value on date $\mathrm{t}$ is $Y_{t}$ and he is trying to determine the optimal time to sell. Additionally, the agent can invest in the market portfolio, which on date $\mathrm{t}$ has a value of $M_{t}$. The market portfolio is traded in a complete market through which the agent can buy complete units or proportions of it.

Suppose that the Real Asset follows a jump-diffusion process described by the following equation:

$$
\frac{d Y_{t}}{Y_{t}}=v d t+\sigma d B_{t}+\xi d q
$$

Let the price of the market portfolio on date $\mathrm{t}, M_{t}$, follow a geometric Brownian motion described by the following:

$$
\frac{d M_{t}}{M_{t}}=\theta \mu d t+\kappa d W_{t}
$$

Let $I_{t}$, the price of a risk-free bond, satisfy:

$$
\frac{d I_{t}}{I_{t}}=
$$

The variables in the above equations are defined as follows: $B$ is a Brownian motion such as $d B_{t}=\rho d W_{t}+$ $\left(1-\rho^{2}\right)^{1 / 2} d Z_{t}$, where $d W$ and $d Z$ are independent uncorrelated Brownian motions, $v$ and $\mu$ are the mean drifts, $\sigma$ and $\kappa$ are the volatilities, $\xi$ is the jump's scale, $r$ is the risk-free rate, $\bar{\rho}$ is a non-negative scalar where $\bar{\rho}=1-\rho^{T} \rho$, and $d q$ is a Poisson random variable with intensity $\lambda$. Furthermore, we define $\varepsilon=(v-r) / \sigma$ and $\delta=(\mu-r) / \kappa$ where $\varepsilon$ and $\delta$ are the Sharpe ratios for the Real Asset and the Market portfolio, respectively.

The agent chooses the amount of wealth invested in $M_{t}, \theta$, and the optimal time to sell the Real Asset, $\tau$, to find his indirect utility of wealth $V_{*}$ through solving the following:

\footnotetext{
${ }^{1}$ See Vollert (2003) for an overview.
} 


$$
V_{*}=V_{*}(x, y)=\sup _{\tau} \sup _{\theta \in A_{\tau}} \mathbb{E}^{x, y} U\left(\tau, M_{\tau}+Y_{\tau}\right)
$$

where $U$ is given by

$$
U(t, x)=e^{-\beta t} \frac{(m+y)^{1-R}}{1-R}
$$

where $\beta$ is the discount rate and is defined by $\beta=(1-R) r+(1-R) \delta^{2} / 2 R$ and $\theta$ is the proportion of the agent's wealth invested in $M_{t} . R$ is the agent's risk aversion. EHH states that such a discount factor insures that $U$ is a super martingale under any $\theta$ and a local martingale under the optimal one resulting in the objective function being horizontally unbiased. The first term in $\beta$ discounts future wealth to current amounts; the second term accounts for the opportunity cost of delaying the sale of the Real Asset.

\section{NUMERICAL ANALYSIS}

We tackle the model above numerically where we simulate the path of the Market portfolio and Real Asset over 30 years, in addition to different values of $\theta$; also for each year we observe $V$ and the corresponding ratio, $W_{t}=$ $Y / M_{t}$. The choice of 30 years is arbitrary and is based on my belief that it represents a sufficient time horizon to observe $V$ and determine the optimal behavior of the agent.

The representative parameter values are as follows: $\delta=0.15, \sigma=0.3, \varepsilon \in\{0.1,0.15,0.2\}, \rho \in\{0,0.25,0.5$, $0.75\}, R \in\{0.25,0.5,0.75\}, r=4 \%, \lambda=0.912$ year, and a normally distributed jump size with parameters $\mathrm{N}(0$, $10 \%$ ). All the above parameters, except for the jump rate and size, have been chosen in accordance with the values used by EHH in their numerical analysis in order to make the analysis more comparable. The parameter values for the jump size and rate are based on the empirical findings of Ball and Torous (1983) and Maheu and McCardy (2004). The jump process numbers mean that the expected number of jumps in the asset's returns is 0.912 per year, with each jump having a return size that is normally distributed with a mean of $0 \%$ and a standard deviation of $10 \%$. The intuition behind choosing the values of $\delta$ and $\varepsilon$ is to observe the optimal exercise ratio of the portfolio as the Sharpe ratio of the Real Asset moves from being less than to greater than that of the Market portfolio. When $\varepsilon<0$, the rational agent will sell the Real Asset immediately as he can achieve a higher expected return by investing the proceeds of the sale of the Real Asset in the risk-free asset.

Proposition I: When $\varepsilon<\rho \delta$, the optimal behavior of the agent is to sell the Real Asset immediately.

This case can be thought of as the Real Asset neither having any return enhancement nor any diversification benefits (i.e., no improvement in the portfolio's risk-return trade). Therefore, the agent has no advantage for holding the Real AssetReal Asset. This can be evidenced by the decline in the agent's utility as shown in Figure 1. The figure shows that the agent holding the Real Asset would find his utility dropping the longer he holds on to it. Given such a result, there is no reason for the agent to hold the Real Asset and he should dispose of it immediately. 


$$
\varepsilon=0.1, \rho=0, R=0.25
$$

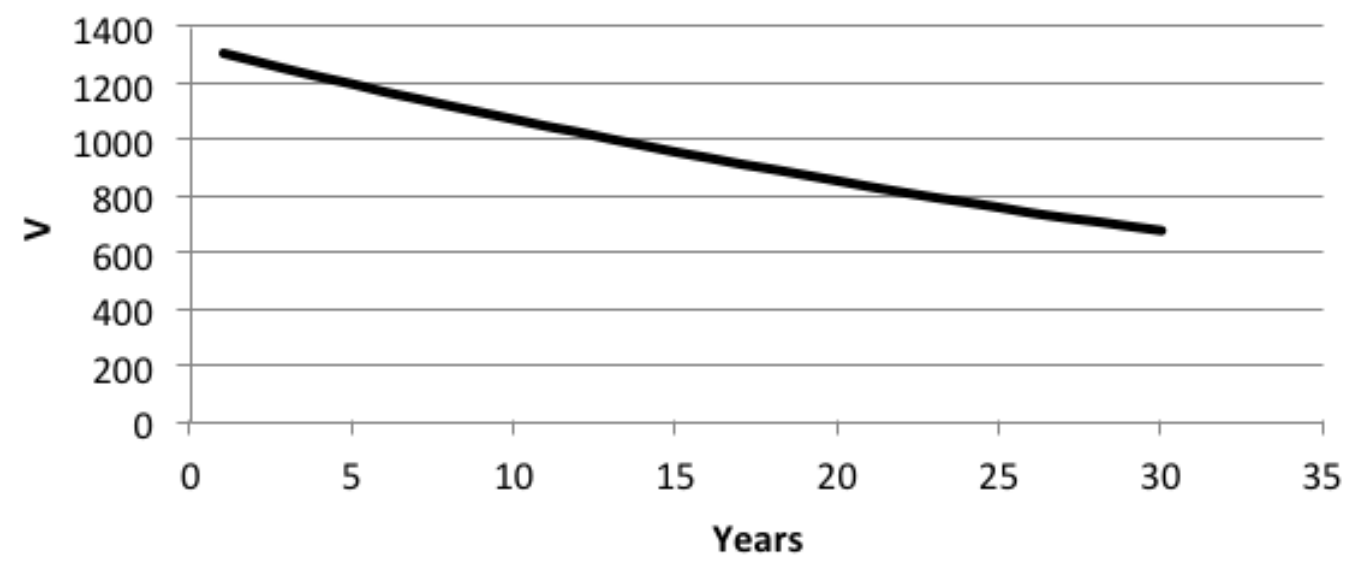

Figure 1: The Agent's Indirect Utility Over Time When $\varepsilon<\rho \delta . \varepsilon=0.01, \rho=0, \mathrm{r}=0.25$

Proposition II: When $\varepsilon>\rho \delta$, the optimal behavior of the agent, depending on parameter values, is either:

(i) Sell the Real Asset immediately, or

(ii) Sell the Real Asset when it reaches the optimal exercise ratio w*, if it existed, or

(iii) Never sell the Real Asset.

Figure 2 below shows the case when, although $\varepsilon>\rho \delta$ (i.e., there are return enhancements and/or diversification benefits to holding the asset), the agent's indirect utility is decreasing over time. Given such a result, it would be proper for the agent to discard the Real Asset immediately to stop his utility from reducing. Such a finding would arise from either of two different sources. This could be driven by the agent's risk aversion and his reluctance to be exposed to the Real Asset's risk. Another possible reason for such a decline in the agent's utility is that the Real Asset has significant levels of non-diversifiable idiosyncratic risk that exceed the gains of holding on to it.

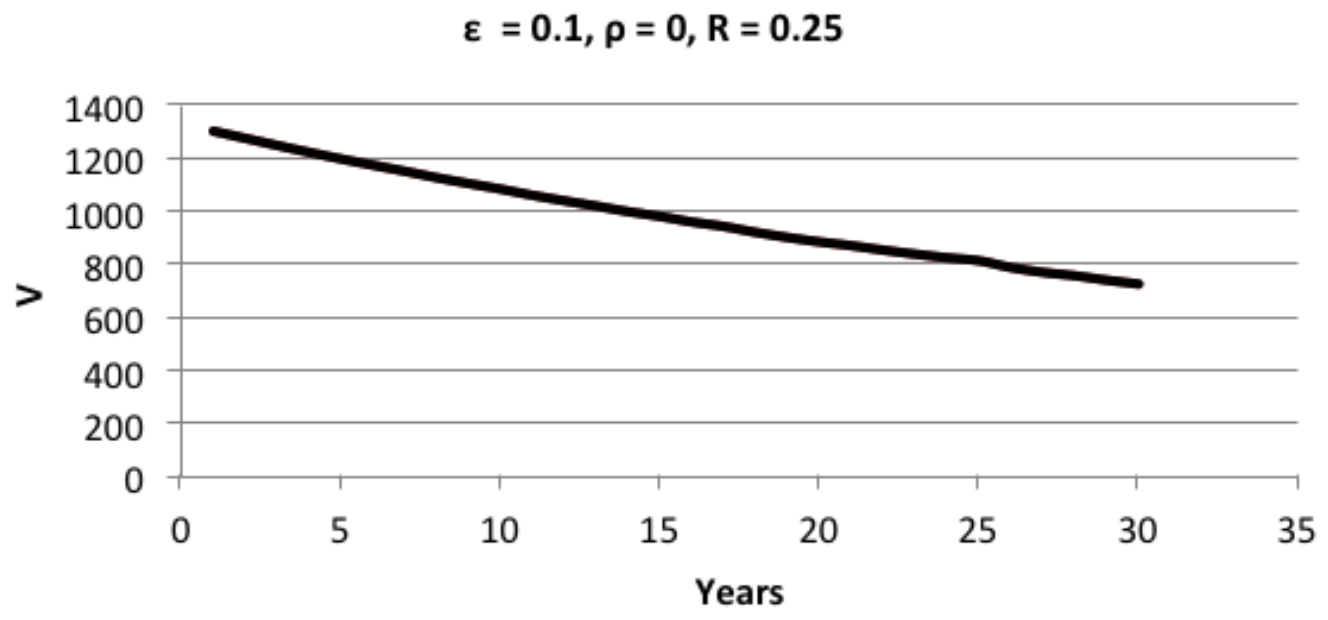

Figure 2: The Agent's Indirect Utility Over Time When $\varepsilon>\rho \delta$ And It Is Optimal To Sell Immediately 
Figure 3 shows the other case where an optimal exercise ratio, $w^{*}$, exists at which the agent should sell the Real Asset. The reasoning behind this is the agent's utility is increasing to a certain point, the optimal exercise ratio $w^{*}$, after which the utility is decreasing; therefore, the agent should sell the Real Asset at $w^{*}$. This implies that, when the Real Asset has return enhancements and/or diversification benefits, the agent is willing to hold on to it until it comprises a certain threshold of his wealth which the agent deems too large; therefore, the agent would dispose of it.



Figure 3: The Agent's Indirect Utility Over Time When $\varepsilon>\rho \delta$ And $w^{*}$ Exists

Figure 4 below represents the third case in which the agent never sells the Real Asset. This finding would be driven by two reasons, depending on the Real Asset's correlation with the market portfolio. At low correlations, the Real Assets would be providing return enhancement and diversification benefits to the agent's portfolio. However, at high correlations, the return enhancement exceeds the loss of diversification benefits driving the agent to optimally never sell the asset. In other words, the Real Asset would have significantly high returns that more than compensate for its non-diversifiable risk. This would result in the agent's portfolio achieving a higher Sharpe ratio by holding the Real Asset than by discarding it, regardless of its size within the portfolio. The figure below shows how the agent's utility increases as he holds the asset over time, resulting in the agent never selling the asset. Although the plot ends at Year 30, the agent's indirect utility will maintain its upward trend. 


$$
\varepsilon=0.2, \rho=0, R=0.75
$$



Figure 4: The Agent's Indirect Utility Over Time When $\varepsilon>\rho \delta$ And It Is Never Optimal To Sell

The previous results have all been in accordance with the findings and results of EHH. This indicates that a Real Asset observing jumps in its motion would lead agents to behave no differently from the case of no jumps.

We now move on to test how changes in the jump process affect the results shown above. The factors that will undergo this sensitivity analysis are the jump rate and the variance of the jump size. This analysis was done through holding everything in the model constant and changing each variable separately.

Proposition III: As the jump rate $\lambda$ increases $w^{*}$ decreases, if it existed.

We observe the behavior of the agent as the jump rate $\lambda$ changes through the following parameter values, 0 , 0.912 , and 2 . The economic reasoning behind the proposition is that an increase in $\lambda$ is translated into an increase in the idiosyncratic risk of the Real Asset. Therefore, a risk-averse agent would hold the Real Asset in a smaller proportion of his wealth as its idiosyncratic risk increased. Figure 5 below shows various $w^{*}$ depending on the jump rates, $\lambda$. Although the plot ends at Year $15, w^{*}$ will not change afterwards.

$$
\varepsilon=0.15, \rho=0.75, R=0.75
$$



Figure 5: $w^{*}$ At Various Jump Rates, $\lambda$ 
Proposition IV: As the jump size variance increases, the change in the optimal behavior of the agent is as follows, depending on parameter values:

(i) If $\mathrm{w}^{*}$ existed previously, the agent either optimally sells the asset immediately or $\mathrm{w}^{*}$ decreases.

(ii) If prior to $\lambda$ 's increase, it was optimal to never sell, the agent will either continue to never sell or $w^{*}$ may arise.

Figure 6 shows Proposition IV.ii, where the optimal path has changed from never selling the Real Asset to having a finite $w^{*}$ at which it is optimal to sell. An increase in the jump size variance translates to an increase in the idiosyncratic risk of the Real Asset. Therefore, a risk-averse agent would now be more likely to dispose of it once it reaches a certain threshold of his wealth at which its risks become too large and not compensated appropriately by its returns.

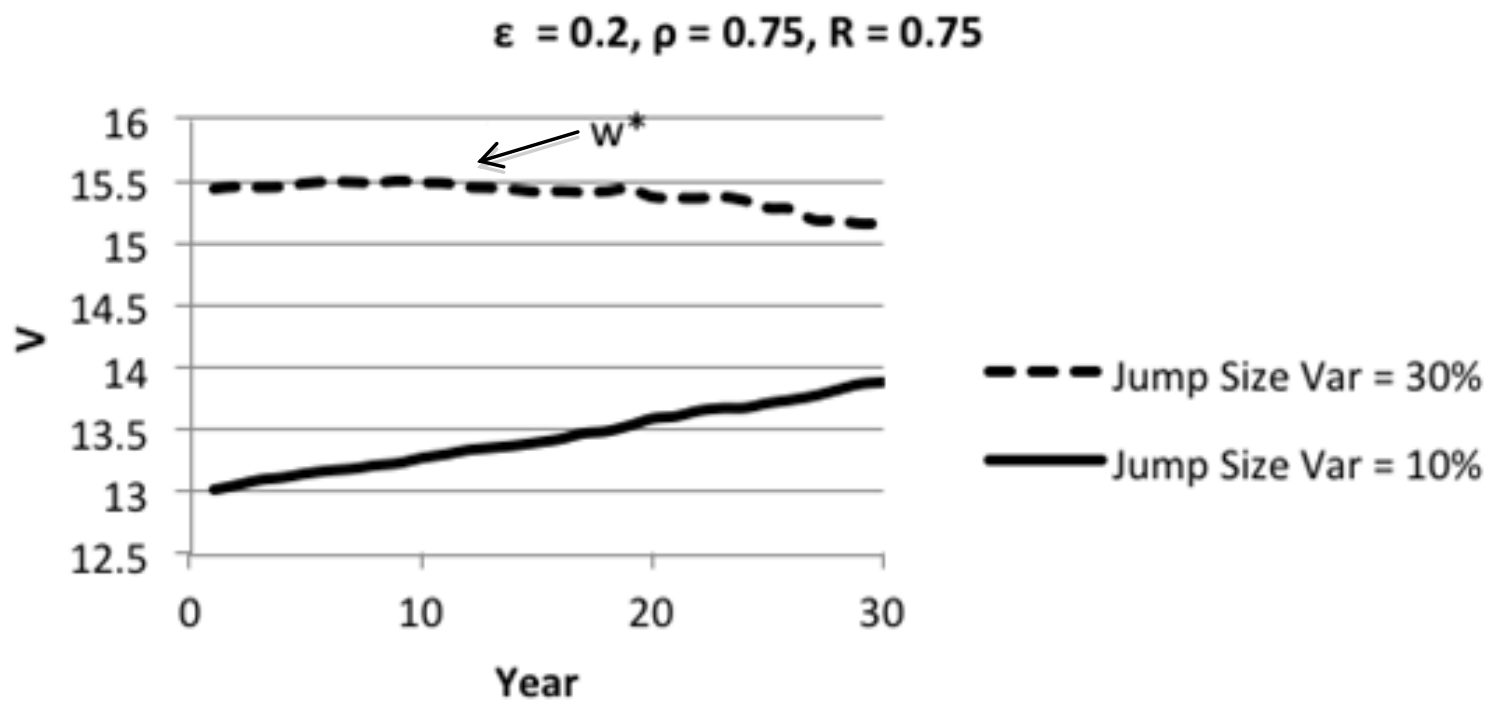

Figure 6: Changes In The Agent's Optimal Behavior As The Jump Size Variance Increases

Figure 7 below represents Proposition IV.i in which the agent had an optimal exercise ratio but as the jump size variance increased, it is no longer optimal for him to hold on to the Real Asset. Selling the asset immediately is observed through the declining indirect utility $\mathrm{V}$ since year 0 ; however, with a smaller jump size variance a $w^{*}$ existed at which afterwards $\mathrm{V}$ starts to decline. The rationale for this is similar to the previous discussed case in which a risk-averse agent would reduce his investment in the Real Asset due to the increase of its idiosyncratic risk. 


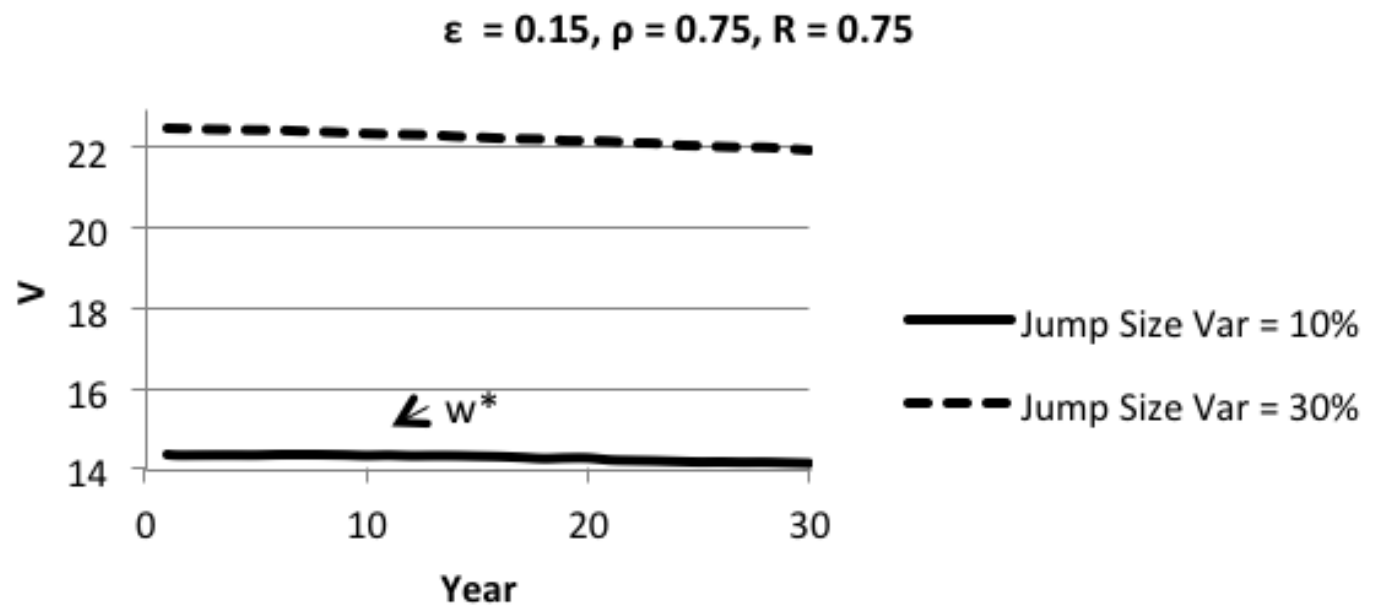

Figure 7: Changes In The Agent's Optimal Behavior As The Jump Size Variance Increases

Figure 8 shows the third case of Proposition IV in which an increase in the jump size variance is accompanied by a decrease in the optimal exercise ratio. Again, the same economic rationale applies.

$$
\varepsilon=0.15, \rho=0, R=0.75
$$



Figure 8: Changes In The Agent's Optimal Behavior As The Jump Size Variance Increases

In summary, an increase in the Real Asset's jump intensity or variance would translate into an increase in the asset's idiosyncratic risk. This, in turn, would lead the risk-averse agent to hold the Real Asset either at smaller proportions of his wealth or to immediately discard the asset.

To provide an example of these findings, let us consider John, a high net-worth individual with a belowaverage risk aversion and a large portfolio in equities, who has recently inherited a large piece of land. Land is a Real Asset that does observe jumps in its price movement. Therefore, John is faced with three choices depending on the risk and return characteristics of the land with respect to his equities portfolio. If the land does not provide him with any return enhancement or diversification benefits (i.e., does not increase his portfolio's Sharpe ratio), he should sell the land immediately. Conversely, if the land does provide a return or risk reduction benefits, John would be faced with two choices. If the improvement in his Sharpe ratio was only modestly significant then John would 
hold the piece of land until it comprised a certain weight in his total portfolio at which he would sell the land. The level of this weight would be a function of his risk aversion. Then, again, if the land improved John's portfolio's Sharpe ratio significantly, he would arrive at the decision to never sell the asset. However, if the land's price behavior were to change and to observe a greater level of jumps, and therefore greater idiosyncratic risk, John would be inclined to hold the piece of land only if it comprised a smaller weight of his assets than he had before.

\section{CONCLUSION}

In this paper, we have extended the work of EHH by adding a jump process to the stochastic movement of the Real Asset and then tackling the problem numerically. The motive behind adding the jump process was to better capture the idiosyncratic risk the agent faces from holding this asset. By more accurately capturing the behavior of the Real Asset, we show that a risk-averse agent would hold the asset at smaller proportions of his wealth, if at all.

The results we have found is that it is not feasible to hold on to the Real Asset when it is neither providing diversification benefits nor return enhancement (i.e., $\varepsilon<\rho \delta$ ). This can be further interpreted as a case where the idiosyncratic risks of holding the asset greatly outweigh any return or diversification benefits. On the other hand, when $\varepsilon>\rho \delta$, the agent will have three different strategies depending on the specific problem parameters. It could be the case that, although $\varepsilon>\rho \delta$, it would remain unfeasible to continue holding the Real Asset due to the agent's risk aversion or to the high idiosyncratic risks that exceed the gains of holding on. The second strategy arises when an optimal exercise ratio exists; in this case, the rational agent will continue to hold on to the Real Asset until it reaches this optimal ratio and will then sell it. It is interesting to note that, as the correlation between the Real Asset and the market portfolio increased, the diversification benefits were reduced and hence, the optimal exercise ratio decreased. The third strategy is when the return on the Real Asset is "too sweet of a deal" to ever sell the asset. These results go hand in hand with what EHH have found in their work.

\section{AUTHOR INFORMATION}

Bader Alhashel is an Assistant professor of Finance \& Financial Institutions. He received his $\mathrm{PhD}$ in Finance from Indiana University - Bloomington. Address: College of Business Administration, Kuwait University, Shuwaikh, Kuwait. Tel: +(965)9944-8300. Email: balhashel@cba.edu.kw

\section{ACKNOWLEDGMENTS}

The author would like to thank Craig W. Holden for his valuable comments and feedback.

\section{REFERENCES}

1. Andersen, L., \& Andreasen, J. (2000). Jump-diffusion processes: Volatility smile fitting and numerical methods for option pricing. Review of Derivatives Research, 4(3), 231-262.

2. Ball, C.A., and Torous, W.N. (1983). A Simplified Jump Process for Common Stock Returns. Journal of Financial and Quantitative Analysis, 18(1), 53-65.

3. Ball, C.A., and Torous, W.N. (1985). On Jumps in Common Stock Prices and Their Impact on Call Option Pricing. Journal of Finance, 40(1), 155-173.

4. Bates, D. S. (1996). Jumps and stochastic volatility: Exchange rate processes implicit in Deutsche Mark options. Review of financial studies, 9(1), 69-107.

5. Cai, N., \& Kou, S. G. (2011). Option pricing under a mixed-exponential jump diffusion model. Management Science, 57(11), 2067-2081.

6. Cont, R., \& Voltchkova, E. (2005). A finite difference scheme for option pricing in jump diffusion and exponential Lévy models. SIAM Journal on Numerical Analysis, 43(4), 1596-1626.

7. Cox, J. C., \& Ross, S. A. (1976). The valuation of options for alternative stochastic processes. Journal of financial economics, 3(1), 145-166.

8. Das, S. R. (2002). The surprise element: jumps in interest rates. Journal of Econometrics, 106(1), 27-65.

9. Duffie, D., Pan, J., \& Singleton, K. (2000). Transform analysis and asset pricing for affine jump-diffusions. Econometrica, 68(6), 1343-1376. 
10. Evans, J.D., Henderson, V. and Hobson, D. (2008). Optimal Timing for an Indivisible Asset Sale. Mathematical Finance, 18(4), 545-567.

11. Henderson, V. (2007). Valuing the option to invest in an incomplete market. Mathematics and Financial Economics, 1(2), 103-128.

12. Kou, S. G. (2002). A jump-diffusion model for option pricing. Management science, 48(8), 1086-1101.

13. Kou, S. G., \& Wang, H. (2003). First passage times of a jump diffusion process.Advances in applied probability, 504-531.

14. Kou, S. G., \& Wang, H. (2004). Option pricing under a double exponential jump diffusion model. Management science, 50(9), 1178-1192.

15. Lee, S. S., \& Mykland, P. A. (2008). Jumps in financial markets: A new nonparametric test and jump dynamics. Review of Financial studies, 21(6), 2535-2563.

16. Maheu, J. M., and McCurdy, T. H. (2004). News Arrival, Jump Dynamics, and Volatility Components for Individual Stock Returns. The Journal of Finance, 59(2), 755-793.

17. McDonald, R., and Siegel, D. (1986). The Value of Waiting to Invest. The Quarterly Journal of Economics, 101(4), 707-727.

18. Merton, R. C. (1976). Option pricing when underlying stock returns are discontinuous. Journal of financial economics, 3(1), 125-144.

19. Press, S. J. (1967). A Compound Events Model for Security Prices. The Journal of Business, 40(3), 317335.

20. Smith, J. E., and Nau, R. F. (1995). Valuing Risky Projects: Option Pricing Theory and Decision Analysis. Management Science, 41(5), 795-816.

21. Vollert, A. (2003). A Stochastic Control Framework for Real Options in Strategic Evaluation. Springer. 